\title{
Statistics of limit root bundles relevant for exact matter spectra of F-theory MSSMs
}

\author{
Martin Bies@ $\oplus^{1,2}$ Mirjam Cvetič $\odot,{ }^{2,1,3}$ and Muyang Liu $\oplus^{2}$ \\ ${ }^{1}$ Department of Mathematics, University of Pennsylvania, Philadelphia, Pennsylvania 19104-6396, USA \\ ${ }^{2}$ Department of Physics and Astronomy, University of Pennsylvania, Philadelphia, \\ Pennsylvania 19104-6396, USA \\ ${ }^{3}$ Center for Applied Mathematics and Theoretical Physics, University of Maribor, Maribor, Slovenia
}

(Received 24 May 2021; accepted 13 July 2021; published 15 September 2021)

\begin{abstract}
In the largest, currently known, class of one quadrillion globally consistent F-theory Standard Models with gauge coupling unification and no chiral exotics, the vectorlike spectra are counted by cohomologies of root bundles. In this work, we apply a previously proposed method to identify toric base threefolds, which are promising to establish F-theory Standard Models with exactly three quark doublets and no vectorlike exotics in this representation. The base spaces in question are obtained from triangulations of 708 polytopes. By studying root bundles on the quark-doublet curve $C_{(\mathbf{3 , 2})_{1 / 6}}$ and employing well-known results about desingularizations of toric K3 surfaces, we derive a triangulation independent lower bound $\check{N}_{P}^{(3)}$ for the number $N_{P}^{(3)}$ of root bundles on $C_{(3,2)_{1 / 6}}$ with exactly three sections. The ratio $\check{N}_{P}^{(3)} / N_{P}$, where $N_{P}$ is the total number of roots on $C_{(\mathbf{3 , 2})_{1 / 6}}$, is largest for base spaces associated with triangulations of the eighth three-dimensional polytope $\Delta_{8}^{\circ}$ in the Kreuzer-Skarke list. For each of these $\mathcal{O}\left(10^{15}\right)$ threefolds, we expect that many root bundles on $C_{(3,2)_{1 / 6}}$ are induced from F-theory gauge potentials and that at least every 3000th root on $C_{(\mathbf{3 , 2})_{1 / 6}}$ has exactly three global sections and thus no exotic vectorlike quark-doublet modes.
\end{abstract}

DOI: 10.1103/PhysRevD.104.L061903

\section{INTRODUCTION}

Like no other framework for quantum gravity, string theory encodes the consistent coupling of gauge dynamics to gravity. Therefore, it is a leading candidate for a unified theory that accounts for all aspects of the observed low energy physics. Enormous efforts have been undertaken to demonstrate the particle spectrum of the Standard Model from string theory. The earliest studies focus on the $E_{8} \times$ $E_{8}$ heterotic string [1-8] and were later extended by intersecting branes models [9-16].

While these compactifications realize the gauge sector and chiral spectrum of the Standard Model, they are limited to the perturbative regime in the string coupling. Typically, they also suffer from vectorlike exotics. The first globally consistent, perturbative minimal supersymmetric standard model (MSSM) constructions are described in [4,5] (see $[17,18]$ for more details).

Published by the American Physical Society under the terms of the Creative Commons Attribution 4.0 International license. Further distribution of this work must maintain attribution to the author(s) and the published article's title, journal citation, and DOI. Funded by SCOAP.
In string compactifications, a significant amount of information is encoded in the geometry of the compactification space. A coherent approach to analyze the relations between geometry and physics is F theory [19-21]. It describes the gauge dynamics of 7-branes including their backreactions to all orders in string coupling. In fourdimensional compactifications, this is achieved by encoding the backreactions in the geometry of a singular elliptically fibered Calabi-Yau fourfold $\pi: Y_{4} \rightarrow B_{3}$. The global consistency conditions of the four-dimensional physics are enforced by studying the geometry of $Y_{4}$, e.g., by a smooth, flat, crepant resolution $\hat{Y}_{4}$.

The chiral spectrum of $4 \mathrm{~d} \mathcal{N}=1$ F-theory compactifications is determined by a background $G_{4}$ flux. This flux is specified by the internal $C_{3}$ profile of the dual M-theory compactification via $G_{4}=d C_{3} \in H^{2,2}\left(\hat{Y}_{4}\right)$, where $H^{2,2}\left(\hat{Y}_{4}\right)$ is the middle vertical fourth cohomology of $\hat{Y}_{4}$. The primary vertical subspace of $G_{4}$ configurations has been studied extensively [22-28]. Applications to globally consistent chiral F-theory models $[26,28-30]$ have lead to the discovery of the largest, currently known, class of one quadrillion globally consistent F-theory Standard Models (QSMs) with gauge coupling unification and no chiral exotics [31]. 
The massless vectorlike spectrum depends not only on $G_{4}$, but also on the $C_{3}$-flat directions. The full gauge information is encoded in Deligne cohomology. In [32-34], F-theory gauge potentials were parametrized by Chow classes, which in turn induce line bundles $L_{\mathbf{R}}$ on the matter curves $C_{\mathbf{R}} \subset B_{3}$. The (vectorlike) zero modes are counted by the cohomologies of these line bundles.

In principle, this approach works for any compactification. Technical limitations arise in explicit geometries due to the intricate complex structure dependence of the cohomologies $h^{i}\left(C_{\mathbf{R}}, L_{\mathbf{R}}\right)$ of the line bundles $L_{\mathbf{R}}$ on the matter curves $C_{\mathbf{R}}$. This dependence was investigated for special examples of F-theory compactifications in [35]. A large data set was generated [36,37] and investigated with data science techniques and completely understood by Brill-Noether theory [38] (cf. [39,40]).

For the QSMs [31] another complication arises. As explained in [41], in these models the line bundles $L_{\mathbf{R}}$ are necessarily root bundles $P_{\mathbf{R}}$, which one may think of as generalizations of spin bundles. Just as spin bundles, there are typically $N_{P}\left(C_{\mathbf{R}}\right) \gg 1$ root bundles on $C_{\mathbf{R}}$. Some of the $N_{P}\left(C_{\mathbf{R}}\right)$ roots stem from F-theory gauge backgrounds which induce the same chiral index but differ in their $\mathrm{C}_{3}$-flat directions. An important task is to find the roots which are induced from F-theory gauge potentials and have cohomologies that define MSSMs.

As a first step, a "bottom-up" analysis was conducted in [41]. This work does not identify exactly which root bundles on $C_{\mathbf{R}}$ are induced from F-theory gauge potentials in the Deligne cohomology. Rather, a systematic study of the cohomologies of all admissible root bundles on $C_{\mathbf{R}}$ has been performed. Except for the Higgs curve, the prime interest are the $N_{P}^{(3)}\left(C_{\mathbf{R}}\right) \leq N_{P}\left(C_{\mathbf{R}}\right)$ roots with exactly three sections. By extending the results in [42], the authors formulated a technique to derive a lower bound $\check{N}_{P}^{(3)}\left(C_{\mathbf{R}}\right)$ to $N_{P}^{(3)}\left(C_{\mathbf{R}}\right)$.

The toric base spaces of the QSMs are obtained from triangulations of 708 polytopes in the Kreuzer-Skarke list [43]. The goal of this paper is to explain that $\breve{N}_{P}^{(3)}\left(C_{\mathbf{R}}\right)$ is independent of the triangulations. We use this observation to identify promising toric threefolds for F-Theory Standard Models without vectorlike exotics on the quark-doublet curve $C_{(\mathbf{3 , 2})_{1 / 6}}$.

In Sec. II we recall the relation of the toric QSM base threefolds and toric $\mathrm{K} 3$ surfaces. By studying limit roots on a nodal curve $C_{(\mathbf{3 , 2})_{1 / 6}}^{*}$ and employing results of resolutions [44-48], we demonstrate in Sec. III that the derived lower bound $\breve{N}_{P}^{(3)}\left(C_{\mathbf{R}}\right)$ is independent of the triangulation. We utilize the Gap4-package QSMExplorer [37] in Sec. III C to compute the ratio $\check{N}_{P}^{(3)} / N_{P}$ for several classes of toric QSM base threefolds. We focus on bases, for which it can be expected that many root bundles on $C_{(\mathbf{3 , 2})_{1 / 6}}$ are "top down" determined by gauge potentials of the F-theory compactification. This points us to the threefolds associated with the $\mathcal{O}\left(10^{15}\right)$ triangulations [49] of the eighth polytope $\Delta_{8}^{\circ}$ in the Kreuzer-Skarke list [43]: At least every 3000th root on $C_{(\mathbf{3 , 2})_{1 / 6}}$ has exactly three global sections and thus no vectorlike exotics.

\section{GENESIS OF THREEFOLD BASES}

Desingularizations of Calabi-Yau (CY) hypersurfaces in toric ambient space are studied in [44]. We focus on CY twofolds, i.e., toric K3 surfaces. Those are associated to three-dimensional, reflexive lattice polytopes $\Delta \subset M_{\mathbb{R}}$ and their polar duals $\Delta^{\circ} \subset N_{\mathbb{R}}$, defined by $\left\langle\Delta, \Delta^{\circ}\right\rangle \geq-1$. Kreuzer and Skarke list all possible three-dimensional polytopes [43]. We consider the $i$ th polytope $\Delta_{i}^{\circ}$ in the Kreuzer-Skarke list as subset of $N_{\mathbb{R}}$.

From a polytope $\Delta \subset M_{\mathbb{R}}$, one can build the normal fan $\Sigma_{\Delta}$. Its ray generators are the facet normals of $\Delta$ and the maximal cones are in one-to-one correspondence to the vertices of $\Delta$. We give a two-dimensional example in Fig. 1. Neither the toric variety $X_{\Delta} \equiv X_{\Sigma_{\Delta}}$ nor the CY hypersurface need be smooth. Resolutions of these CY hypersurfaces were introduced in [44] as maximal projective crepant partial (MPCP) desingularizations. Equivalently, [46] refers to such desingularizations as maximal projective subdivisions of the normal fan.

To find MPCPs, we note that a refinement of the normal fan $\Sigma_{\Delta}$ by ray generators corresponding to lattice points of $\Delta^{\circ}$ is crepant. We can therefore consider refinements $\Sigma(T) \rightarrow \Sigma_{\Delta}$ where $\Sigma(T)$ is associated to a fine, regular, star triangulation (FRST) of the lattice polytope $\Delta^{\circ}$. We recall that star means that every simplex in the triangulation contains the origin, fine ensures that every lattice point of $\Delta^{\circ}$ is used as ray generator, and that regular implies that $X_{\Sigma(T)}$ is projective. Together, this implies that $\Sigma(T)$ defines a maximal projective refinement of $\Sigma_{\Delta}$. This is illustrated in Fig. 1.

In our applications to toric $\mathrm{K} 3$ surfaces, $X_{\Sigma(T)}$ is guaranteed to be smooth. This is because a maximal projective subdivision of $\Sigma_{\Delta}$ then constructs a three-dimensional Gorenstein orbifold with terminal singularities [46] which

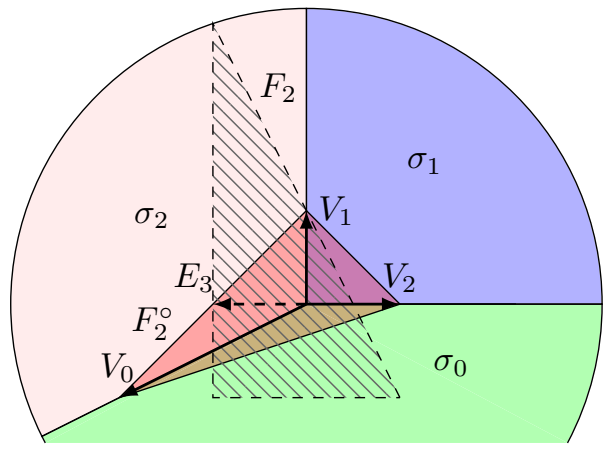

FIG. 1. MPCP of $F_{2}^{\circ}=\operatorname{Conv}\left(e_{1}, e_{2},-2 e_{1}-e_{2}\right) \subset N_{\mathbb{R}}$ refines normal fan $\Sigma_{F_{2}}$ of polytope $F_{2} \subseteq M_{\mathbb{R}}$ by $E_{3}$. 

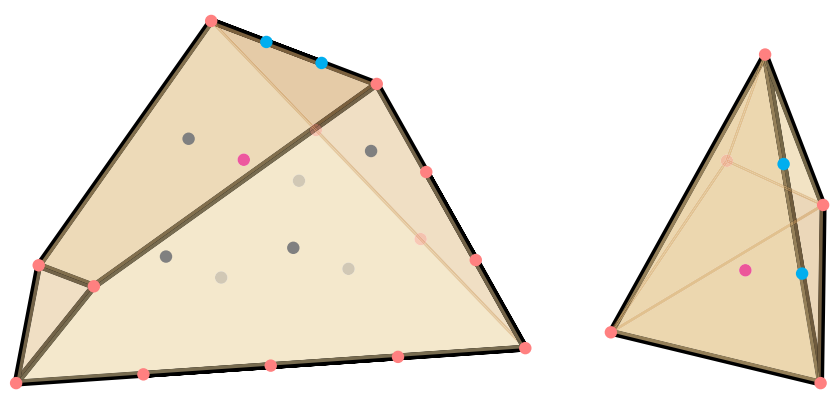

FIG. 2. $\quad \Delta_{52}^{\circ} \subset N_{\mathbb{R}}$ on the left and $\Delta_{52} \subseteq M_{\mathbb{R}}$ on the right [43]. The magenta point is the origin. The generic K3 surface meets trivially with gray divisors, in an irreducible curve with the pinks and in finite families of $\mathbb{P}^{1} \mathrm{~s}$ with the cyans.

must be smooth by proposition 11.4.19 in [50] (see also [44]).

Of the 4319 polytopes in [43], 708 lead to toric threefolds with $\bar{K}_{X_{\Sigma}(T)}^{3} \in\{6,10,18,30\}$. Those are the base spaces for the QSMs [31], in which the gauge divisors are K3 surfaces. This leads to gauge coupling unification. In the rest of this paper, we reserve the symbol $B_{3}\left(\Delta^{\circ}\right)$ for the family of all toric threefolds obtained from FRSTs of the polytope $\Delta^{\circ}$. Our standing example will be the spaces obtained from $\Delta_{52}^{\circ}$ displayed in Fig. 2.

\section{TRIANGULATION INDEPENDENCE}

\section{A. Dual graph}

We will demonstrate that the dual graph of the nodal quark-doublet curve $C_{(\mathbf{3 , 2})_{1 / 6}}^{\cdot}$ introduced in [41] is identical for all threefolds $B_{3}\left(\Delta^{\circ}\right)$ obtained from FRSTs of $\Delta^{\circ}$. Hence, this dual graph only depends on $\Delta^{\circ}$.

The homogeneous coordinates of $X_{\Sigma(T)}$ correspond to the lattice points of $\Delta^{\circ}$. A coordinate associated to a facet interior point is denoted by $z_{c}$. For a lattice point in the interior of an edge $\Theta^{\circ} \subset \Delta^{\circ}$, two facets $F_{1}, F_{2}$ of $\Delta^{\circ}$ meet at $\Theta^{\circ}$. We notice that they are dual to vertices $m_{1}, m_{2} \in \Delta$, and the dual edge $\Theta$ is the edge connecting $m_{1}$ and $m_{2}$. If $\Theta$ has interior points, we denote the homogeneous coordinate as $y_{b}$ and otherwise by $x_{a}$. We mark these distinct types of lattice points in different colors in Fig. 2. The nodal curve $C_{(3,2)_{1 / 6}}^{*}[41]$ is given by

$$
C_{(\mathbf{3 , 2})_{1 / 6}}^{\cdot}=\cup_{a \in A} V\left(x_{a}, s_{9}\right) \cup \underset{b \in B}{\cup} V\left(y_{b}, s_{9}\right) \cup \underset{c \in C}{\cup} V\left(z_{c}, s_{9}\right),
$$

where $s_{9}$ is a generic section of $\bar{K}_{X_{\Sigma(T)}}$. The rational behind this classification is that $V\left(x_{a}, s_{9}\right)$ is irreducible, $V\left(y_{b}, s_{9}\right)$ a finite collection of $\mathbb{P}^{1} \mathrm{~s}$ and $V\left(z_{c}, s_{9}\right)=\varnothing$.

We begin with $V\left(z_{c}, s_{9}\right)=\varnothing$, which was originally proven in [44,46] (see also [51]). Since $X_{\Sigma(T)}$ is associated to a refinement of $\Sigma_{\Delta}$, there is a toric morphism $\varphi: X_{\Sigma(T)} \rightarrow X_{\Delta}$. By construction, this blowdown morphism is crepant and $V\left(z_{c}\right)$ is blown down to a point, so that it does not intersect generic members of $\left|\bar{K}_{X_{\Delta}}\right|$. Since $\varphi$ is crepant and birational, $V\left(z_{c}\right)$ does therefore not intersect generic members of $\bar{K}_{\Sigma(T)}$, i.e., $V\left(z_{c}, s_{9}\right)=\varnothing$. Hence, only the pink and cyan lattice points in Fig. 2 matter.

To see that $V\left(y_{b}, s_{9}\right)$ is reducible, we compute its selfintersection in the K3 surface $V\left(s_{9}\right)$. More generally, topological intersection numbers capture properties of $C_{(3,2)_{1 / 6}}^{0}$. For example, a curve component $C_{i}$ associated to $D_{i} \in \operatorname{Div}_{T}\left(X_{\Sigma(T)}\right)$ has arithmetic genus $g\left(C_{i}\right)$ with $2 g\left(C_{i}\right)-2=D_{i}^{2} \bar{K}_{X_{\Sigma(T)}}$. Similarly, the topological intersection of $C_{i}$ and $C_{j}$ is $D_{i} D_{j} \bar{K}_{X_{\Sigma(T)}}$. From the original work [45] (see also [47]), it follows that these intersection numbers are counted by properties of $\left(\Delta^{\circ}, \Delta\right)$ and are thus independent of the FRST. Let us restate this result.

Proposition: Let $D_{1}, D_{2} \in \operatorname{Div}_{T}\left(X_{\Sigma(T)}\right)$ be two distinct divisors corresponding to lattice points $v_{1}, v_{2} \in \Delta^{\circ}$. If $v_{1}, v_{2}$ are not contained in an edge $\Theta^{\circ} \subset \Delta^{\circ}$, then $D_{1} D_{2} \bar{K}_{X_{\Sigma(T)}}=0$. Otherwise, $D_{1} D_{2} \bar{K}_{X_{\Sigma(T)}}=1+l^{\prime}(\Theta)$, where $l^{\prime}(\Theta)$ is the number of interior lattice points on the dual edge $\Theta$.

Proof.-Consider a triangulation $T$ of $\Delta^{\circ}$. Then the triple intersection among divisors $D_{1}, D_{2}$, and $\bar{K}_{X_{\Sigma(T)}}$ vanishes unless $v_{1}, v_{2}$ belong to two triangles in $T$, which we denote as $v_{1} v_{2} v_{3}$ and $v_{1} v_{2} v_{4}$. It follows that $D_{1} D_{2} D_{i}=0$ if $i \notin$ $\{1,2,3,4\}$ and $D_{1} D_{2} D_{3}=D_{1} D_{2} D_{4}=1$. Hence,

$$
D_{1} D_{2} \bar{K}_{X_{\Sigma(T)}}=D_{1} D_{2}\left(D_{1}+D_{2}+D_{3}+D_{4}\right) \text {. }
$$

The affine span of $v_{1}, v_{2}, v_{3}$ contains a facet $F_{3}$ of $\Delta^{\circ}$. The dual of $F_{3}$ is a vertex $m_{3} \in \Delta$ with $\left\langle m_{3}, v_{i}\right\rangle=-1$. Let $N=\operatorname{rk}\left(\operatorname{Div}_{T}\left(X_{\Sigma(T)}\right)\right)$, then $0 \sim \sum_{i=1}^{N}\left\langle m_{3}, v_{i}\right\rangle D_{i}$ and $D_{1}+D_{2}+D_{3} \sim\left\langle m_{3}, v_{4}\right\rangle D_{4}+S$, where $S$ satisfies $S D_{1} D_{2}=0$. By substituting this back into Eq. (2) we find $D_{1} D_{2} \bar{K}_{X_{\Sigma(T)}}=1+\left\langle m_{3}, v_{4}\right\rangle$. If $v_{1}, v_{2}$ are not contained in an edge $\Theta^{\circ} \subset \Delta^{\circ}$, then $v_{4} \in F_{3},\left\langle m_{3}, v_{4}\right\rangle=-1$ and $D_{1} D_{2} \bar{K}_{X_{\Sigma(T)}}=0$.

Conversely, if $v_{1}, v_{2} \in \Theta^{\circ} \subseteq \Delta^{\circ}$, then $v_{1} v_{2} v_{4}$ is contained in a facet $F_{4} \neq F_{3}$ of $\Delta^{\circ}$ with dual vertex $m_{4} \in \Delta$. The dual edge $\Theta$ from $m_{3}$ to $m_{4}$ only depends on $v_{1}, v_{2}$ but not the triangulation $T$. We now compare the number of interior lattice points $l^{\prime}(\Theta)$ on $\Theta$ to

$$
I_{12}=D_{1} D_{2} \bar{K}_{X_{\Sigma(T)}}=1+\left\langle m_{3}, v_{4}\right\rangle
$$

$v_{1}, v_{2}, v_{4}$ generate $N_{\mathbb{R}}$. Therefore, $m \in M_{\mathbb{R}}$ is a lattice point iff $\left\langle m, v_{1}\right\rangle,\left\langle m, v_{2}\right\rangle$ and $\left\langle m, v_{4}\right\rangle$ are integers. Hence, lattice points on $\Theta$ are $m(k)=m_{3}+\left(\frac{1+k}{I_{12}}\right) \cdot\left(m_{4}-m_{3}\right)$, where $k \in\left\{-1, \ldots, I_{12}\right\}$. Therefore, $l^{\prime}(\Theta)=I_{12}-1$.

We extend this to the arithmetic genera by restating another result from [45]. 
Corollary: Let $D_{1} \in \mathrm{Cl}\left(X_{\Sigma(T)}\right)$ be the divisor associated to the lattice point $v_{1} \in \Delta^{\circ}$. Then $D_{1}^{2} \bar{K}_{X_{\Sigma(T)}}$ is independent of triangulations of $\Delta^{\circ}$. Furthermore, if $v_{1}$ is an interior point of an edge $\Theta^{\circ} \subset \Delta^{\circ}$, then $D_{1}^{2} \bar{K}_{X_{\Sigma(T)}}=$ $-2-2 l^{\prime}(\Theta)$.

Proof.-We consider a facet $F \subset \Delta^{\circ}$ with $v_{1} \in F$. The dual vertex $m \in \Delta \in M_{\mathbb{R}}$ establishes $0 \sim \sum_{i=1}^{N}\left\langle m, v_{i}\right\rangle D_{i}$ and hence $D_{1}^{2} \bar{K}_{X_{\Sigma(T)}}=\sum_{i=2}^{N}\left\langle m, v_{i}\right\rangle D_{1} D_{i} \bar{K}_{X_{\Sigma(T)}}$, which is independent of FRSTs of $\Delta^{\circ}$ by the preceding proposition.

Next, assume that $v_{1}$ in an interior point of an edge $\Theta^{\circ} \subset \Delta^{\circ}$ and denote its neighbors along $\Theta^{\circ}$ by $v_{2}, v_{3}$. The associated divisors $D_{2}, D_{3}$ are the only divisors with nonzero $D_{1} D_{2} \bar{K}_{X_{\Sigma(T)}}, D_{1} D_{3} \bar{K}_{X_{\Sigma(T)}}$. Note that $v_{1}, v_{2}, v_{3}$ are contained in a facet of $\Delta^{\circ}$, whose dual vertex $m \in \Delta$ establishes $\quad D_{1} \sim-D_{2}-D_{3}+S$ with $D_{1} S \bar{K}_{X_{\Sigma(T)}}=0$. Hence, $D_{1}^{2} \bar{K}_{X_{\Sigma(T)}}=-2-2 l^{\prime}(\Theta)$.

$V\left(y_{b}, s_{9}\right)$ corresponds to $v_{b} \in \Theta^{\circ} \subset \Delta^{\circ}$ with $l^{\prime}(\Theta)>0$. Hence $D_{b}^{2} \cdot \bar{K}_{X_{\Sigma(T)}}=-2\left(l^{\prime}(\Theta)+1\right)<-2$ and $V\left(y_{b}, s_{9}\right)$ is reducible into a collection of $l^{\prime}(\Theta)+1$ nonintersecting and smooth $\mathbb{P}^{1} \mathrm{~s}[44,45]$.

Finally, let us turn to the components $V\left(x_{a}, s_{9}\right)$. A subset of these components is associated to lattice points $v_{a} \in \Theta^{\circ}$ such that $l^{\prime}(\Theta)=0$. By the previous result, these components are irreducible and smooth. The remaining $V\left(x_{a}, s_{9}\right)$ 's are associated to the vertices of $\Delta^{\circ}$. These components are smooth and irreducible by [44].

\section{B. Computing the lower bound $\check{N}_{P}^{(3)}$}

We have established that in every space in $B_{3}\left(\Delta^{\circ}\right)$, $C_{(3,2)_{1 / 6}}^{*}$ consists of the same components $C_{i}$ with the same topological properties. Therefore, the dual graph $G\left(C_{(3,2)_{1 / 6}}^{*}\right)$, in which components are vertices and intersections are edges, only depends on $\Delta^{\circ}$. We recall from [41], that on $C_{(\mathbf{3 , 2})_{1 / 6}}^{\cdot}$ we look for limit roots $P_{(\mathbf{3 , 2})_{1 / 6}}^{\cdot}$ with

$$
\left(P_{(3,2)_{1 / 6}}^{\cdot}\right)^{\otimes 2 \bar{K}_{X_{\Sigma(T)}}^{3}}=\left(\left.\bar{K}_{X_{\Sigma(T)}}\right|_{C_{(3,2)_{1 / 6}}}\right)^{\otimes\left(6+\bar{K}_{X_{\Sigma(T)}^{3}}^{3}\right.} .
$$

Such roots are specified by weight assignments to $G\left(C_{(3,2)_{1 / 6}}^{\cdot}\right)$, constrained by $\left(6+\bar{K}_{X_{\Sigma(T)}}^{3}\right)$ times the degree of $\left.\bar{K}_{X_{\Sigma(T)} \mid}\right|_{C_{i}}$. For $V\left(x_{a}, s_{9}\right),\left.\bar{K}_{X_{\Sigma(T)}}\right|_{C_{i}}$ has degree $D_{a} \bar{K}_{X_{\Sigma(T)}}^{2}$. For the irreducible components of $V\left(y_{b}, s_{9}\right)$, this degree is $D_{b} \bar{K}_{X_{\Sigma(T)}}^{2} /\left(l^{\prime}(\Theta)+1\right)$. Since $\bar{K}_{X_{\Sigma(T)}}=\sum_{i=1}^{N} D_{i}$, we have $D \bar{K}_{X_{\Sigma(T)}}^{2}=\sum_{i=1}^{N} D D_{i} \bar{K}_{X_{\Sigma(T)}}$ and by the results of [45] restated in the previous section, these degrees are FRST invariant. Similarly, $\bar{K}_{X_{\Sigma(T)}}^{3}$ is FRST invariant. Consequently, the data that specifies the limit roots on $C_{(3,2)_{1 / 6}}^{\cdot}$ depends only on $\Delta^{\circ}$. By extending the techniques of [41], we can thus compute an FRST-invariant lower bound $\check{N}_{P}^{(3)}$ to the number of root bundles on $C_{(\mathbf{3 , 2})_{1 / 6}}$ with exactly three global sections.

We illustrate our strategy with $\Delta_{52}^{\circ}$ in Fig. 2. Its FRSTs give toric threefolds with $h^{21}\left(\hat{Y}_{4}\right)=7>6=g\left(C_{(\mathbf{3 , 2})_{1 / 6}}\right)$ and $\bar{K}_{B_{3}}{ }^{3}=10$. The dual graph $G\left(C_{(\mathbf{3 , 2})_{1 / 6}}\right)$ is

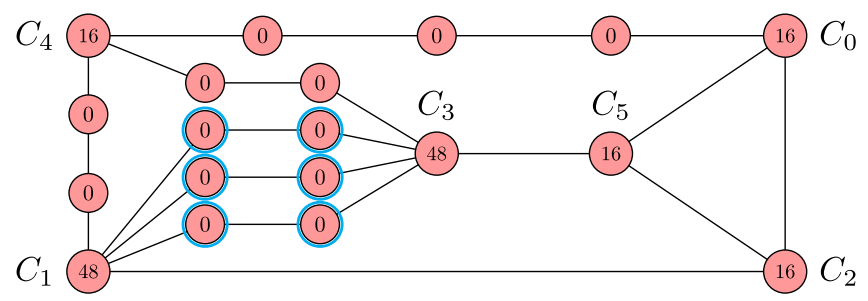

The labels inside the nodes are the degree of $16 \cdot \bar{K}_{C_{(3,2)_{1 / 6}}}$ on the components $C_{i}$. To find the $\left(2 \bar{K}_{X_{\Sigma(T)}}^{3}\right)$ th roots, we place weights $u_{i}, v_{i} \in\left\{1,2, \ldots, 2 \bar{K}_{X_{\Sigma(T)}}^{3}-1\right\}$ along each edge in $G\left(C_{(3,2)_{1 / 6}}\right)$ subject to the following rules (see [41,42] for details):

(1) Along each edge, the sum of weights is $2 \bar{K}_{X_{\Sigma(T)}}^{3}$.

(2) At each node $C_{i}$, the sum of weights equals $\left(6+\bar{K}_{X_{\Sigma(T)}}^{3}\right)$-times $D_{i} \bar{K}_{X_{\Sigma(T)}}^{2}$ modulo $2 \bar{K}_{X_{\Sigma(T)}}^{3}$.

The number of possible weight assignments grows rapidly with the complexity of the dual graph. To speed up the counting, it is possible to replace $\left.G\left(C_{(3,2)}\right)_{\mathrm{i} / 6}\right)$ with a simplified graph. We remove components $C_{i}$ which are connected to exactly two other components and have $D_{i} \bar{K}_{X_{\Sigma(T)}}^{2}=0$. We are thus looking at transitions:

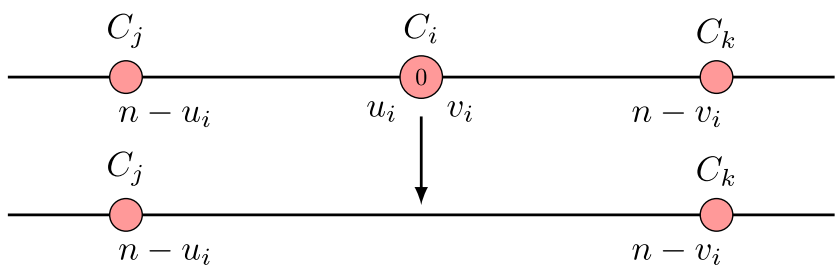

To see that this does not change the lower bound $\check{N}_{P}^{(3)}$, let us focus on $n$th roots. Then $1 \leq u_{i}, v_{i} \leq n-1$. For given $u_{i}$ the weight $v_{i}$ is uniquely fixed as $v_{i}=n-u_{i}$. Since, $D_{i} \bar{K}_{X_{\Sigma(T)}}^{2}=0$, the resulting root on $C_{i}$ has degree -1 and supports no nontrivial sections (cf. [41,42]). Conversely, given the diagram at the bottom, we can reconstruct the original diagram by noting that $v_{i}=n-u_{i}$. For $\Delta_{52}^{\circ}$, this leads to the simplified graph:

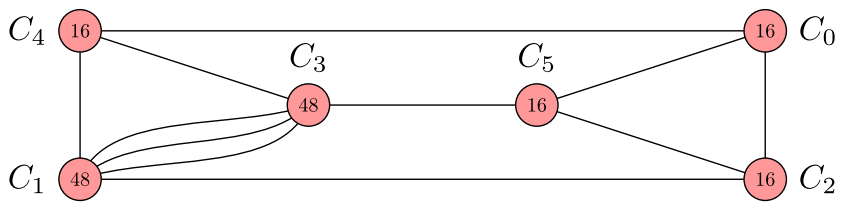


The algorithmic task of finding all weight assignments and counting the associated limit roots can be conducted with a computer implementation. The algorithms employed for this work are available in the Gap4-package QSMExplorer, as part of the ToricVarieties_project [37]. On the computer plesken. mathematik. uni-siegen. de, our algorithm completes for $\Delta_{51}^{\circ}$ in roughly three minutes and finds $\check{N}_{P}^{(3)}=34.980 .351$. This number is to be compared to the total number of root bundles $N_{P}=20^{12}$ on this $g=6$ curve. Hence, at least every $1.2 \times 10^{8}$ th root on $C_{(\mathbf{3 , 2})_{1 / 6}}$ has exactly three global sections.

\section{Towards favorable F-theory base spaces}

We extend this analysis to several classes $B_{3}\left(\Delta^{\circ}\right)$. Among the 708 polytopes, we focus on base spaces for which it can be expected that many roots stem from inequivalent F-theory gauge potentials in the Deligne cohomology $H_{D}^{4}\left(\hat{Y}_{4}, \mathbb{Z}(2)\right)$, i.e., gauge potentials which induce the same chiral index but differ in their $C_{3}$-flat directions. In the fourfold geometry $\hat{Y}_{4}$, these $C_{3}$-flat directions are described by the intermediate Jacobian $J^{2}\left(\hat{Y}_{4}\right)$. Since $h^{3,0}\left(\hat{Y}_{4}\right)=0$, we have (see [32] and references therein) $J^{2}\left(\hat{Y}_{4}\right)=H^{2,1}\left(\hat{Y}_{4}\right) / H^{3}\left(\hat{Y}_{4}, \mathbb{Z}\right) \quad$ and $\operatorname{dim}_{\mathbb{C}}\left(J^{2}\left(\hat{Y}_{4}\right)\right)=h^{2,1}\left(\hat{Y}_{4}\right)$. In particular, if a gauge potential in $H_{D}^{4}\left(\hat{Y}_{4}, \mathbb{Z}(2)\right)$ admits $\left(2 \bar{K}_{X_{\Sigma(T)}}^{3}\right)$ th roots, then it admits $\left(2 \bar{K}_{X_{\Sigma(T)}}^{3}\right)^{2 h^{2,1}\left(\hat{Y}_{4}\right)}$ roots. On the genus $g$ curve $C_{(\mathbf{3 , 2})_{1 / 6}}$, we find $\left(2 \bar{K}_{X_{\Sigma(T)}}^{3}\right)^{2 g}$ roots. In general, only a subset of these root bundles are induced from F-theory gauge potentials. Currently, our algebraic control is insufficient to identify these physical roots explicitly. We focus on threefolds $B_{3}$ for which there are at least as many roots in Deligne cohomology as root bundles on the matter curve, so that statistically speaking it is likely that many, maybe even all, of the $\left(2 \bar{K}_{X_{\Sigma(T)}}^{3}\right)^{2 g}$ root bundles are physical. For example, the base spaces $B_{3}\left(\Delta_{8}^{\circ}\right)$ have $\bar{K}_{B_{3}}{ }^{3}=6, h^{2,1}\left(\hat{Y}_{4}\right)=16, g=$ 4 and $12^{32}$ gauge potentials outnumber $12^{8}$ root bundles. Consequently, we focus on threefolds with $h^{2,1}\left(\hat{Y}_{4}\right) \geq g$ and notice

\begin{tabular}{lccr}
\hline $\bar{K}_{B_{3}}{ }^{3}$ & \# Polytopes & $h^{21}\left(\hat{Y}_{4}\right)$ & $g$ \\
\hline 6 & 7 & $\{8,9,10,12,16\}$ & 4 \\
10 & 54 & $\{2,3, \ldots, 11\} \cup\{13\}$ & 6 \\
18 & 373 & $\{0,1, \ldots, 12\}$ & 10 \\
30 & 274 & $\{0,1, \ldots, 9\}$ & 16 \\
\hline
\end{tabular}

All 7 polytopes with $\bar{K}_{B_{3}}{ }^{3}=6$ satisfy this necessary condition. Their triangulations give at least $50 \%$ of the QSM threefold base spaces [49]. In addition, 27 polytopes with $\bar{K}_{B_{3}}{ }^{3}=10$ and three with $\bar{K}_{B_{3}}{ }^{3}=18$ have this property. For the $\bar{K}_{B_{3}}{ }^{3}=10$ polytope $\Delta_{14}^{\circ}$ and the three $\bar{K}_{B_{3}}{ }^{3}=18$ polytopes $\Delta_{72}^{\circ}, \Delta_{229}^{\circ}$ and $\Delta_{527}^{\circ}$, the quark-doublet curve has a component with genus larger than 1 . Hence, for
TABLE I. $\quad \check{N}_{P}^{(3)}$ for 33 QSM polytopes with $h^{21}\left(\hat{Y}_{4}\right) \geq g$.

\begin{tabular}{|c|c|c|c|c|c|}
\hline \multicolumn{6}{|c|}{$\bar{K}_{B_{3}}{ }^{3}=6: N_{P}\left(C_{(\mathbf{3}, 2)_{1 / 6}}\right)=12^{8}$} \\
\hline & $\check{N}_{P}^{(3)}$ & $N_{P} / \check{N}_{P}^{(3)}$ & & $\check{N}_{P}^{(3)}$ & $N_{P} / \check{N}_{P}^{(3)}$ \\
\hline 8 & 142560 & $3.0 \times 10^{3}$ & & 10 & $4.8 \times 10^{4}$ \\
\hline 4 & & & & & \\
\hline$\Delta_{134}^{\circ}$ & 00 & $4.3 \times 10^{4}$ & $\Delta_{236}^{\circ}$ & 8910 & $1.8 \times 10^{4}$ \\
\hline 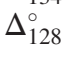 & 8910 & $4.8 \times 10^{4}$ & & & \\
\hline \multicolumn{6}{|c|}{$\bar{K}_{B_{3}}{ }^{3}=10: N_{P}\left(C_{(\mathbf{3}, \mathbf{2})_{1 / 6}}\right)=20^{12}$} \\
\hline & $\check{N}_{P}^{(3)}$ & $N_{P} / \check{N}_{P}^{(3)}$ & & $\check{N}_{P}^{(3)}$ & $N_{P} / \check{N}_{P}^{(3)}$ \\
\hline & 1.680 .888 & $5.2 \times 10^{6}$ & & 2.85 & $1.2 \times 10^{8}$ \\
\hline 0 & 38.662 .983 & $0^{6}$ & $\Delta_{41}^{\circ}$ & 2.85 & $1.2 \times 10^{8}$ \\
\hline$\Delta_{272}^{\circ}$ & 736.011 .640 & $5.6 \times 10^{6}$ & $\Delta_{838}^{\circ+1}$ & 2.84 & $1.2 \times 10^{8}$ \\
\hline$\Delta_{274}^{\circ}$ & 736.011 .640 & $5.6 \times 10^{6}$ & $\Delta_{782}^{\circ}$ & 32.84 & $1.2 \times 10^{8}$ \\
\hline$\Delta_{387}^{\circ}$ & 733.798 .30 & $5.6 \times 10^{6}$ & $\Delta_{377}^{\circ}$ & 30.84 & $1.3 \times 10^{8}$ \\
\hline$\Delta_{798}^{\circ}$ & 690.950 .608 & $5.9 \times 10^{6}$ & $\Delta_{499}^{\circ}$ & 30.846 .440 & $1.3 \times 10^{8}$ \\
\hline$\Delta_{808}^{\circ}$ & 690.950 .608 & $5.9 \times 10^{6}$ & $\Delta_{503}^{\circ}$ & 30.846 .440 & $1.3 \times 10^{8}$ \\
\hline$\Delta_{810}^{\circ}$ & 690.950 .608 & $5.9 \times 10^{6}$ & $\Delta_{1348}^{\circ}$ & 30.845 .702 & $1.3 \times 10^{8}$ \\
\hline$\Delta_{812}^{\circ}$ & 690.950 .608 & $5.9 \times 10^{6}$ & $\Delta_{882}^{\circ}$ & 30.840 .098 & $1.3 \times 10^{8}$ \\
\hline$\Delta_{254}^{\circ}$ & 35.004 .914 & $1.2 \times 10^{8}$ & $\Delta_{1340}^{\circ}$ & 28.954 .543 & $1.4 \times 10^{8}$ \\
\hline$\Delta_{52}^{\circ}$ & & $1.2 \times 10^{8}$ & $\Delta_{1879}^{\circ}$ & 28.950 .852 & $1.4 \times 10^{8}$ \\
\hline$\Delta_{30}^{\circ}$ & 682 & $1.2 \times 10^{8}$ & $\Delta_{1384}^{\circ}$ & 27.178 .020 & $1.5 \times 10^{8}$ \\
\hline 100 & 32.860 .461 & $1.2 \times 10^{8}$ & $\Delta_{856}^{\circ}$ & 22.807 .749 & $1.8 \times 10^{8}$ \\
\hline
\end{tabular}

these spaces, the counting procedure introduced in [41] does not apply. However, for the remaining 33 polytopes, our computer implementation finds $\check{N}_{P}^{(3)}\left(C_{(\mathbf{3 , 2})_{1 / 6}}\right)$ within a few minutes. These results are listed in Table I.

Among these 33 polytopes, the ratio $\check{N}_{P}^{(3)} / N_{P}$ is largest for $\Delta_{8}^{\circ}$. In addition, within the QSMs, base spaces obtained from FRSTs of $\Delta_{8}^{\circ}$ have the maximal $16=h^{21}\left(\hat{Y}_{4}\right)$ and the minimal $g=4$. In this sense, they most positively satisfy the necessary condition for a top-down origin of at least some of the root bundles. In this sense, these $\mathcal{O}\left(10^{15}\right)$ toric base threefolds [49] are currently the most promising candidates to establish an F-theory Standard Model with exactly three quark doublets and no vectorlike exotics in this representation.

\section{DISCUSSION AND OUTLOOK}

A construction of one quadrillion globally consistent Ftheory Standard Models (QSMs) with gauge coupling unification and no chiral exotics was presented in [31]. In this work, we apply the techniques introduced in [41] systematically to the toric QSM base threefolds. Our goal is to identify toric base spaces, which are promising candidates to establish F-theory Standard Models with exactly three quark doublets and no vectorlike exotics in this representation. 
We recall that vectorlike spectra are counted by cohomologies of line bundles $L_{\mathbf{R}}$ on the matter curves $C_{\mathbf{R}}$. In [41], it was argued that these bundles must necessarily be root bundles. For instance, on the quark-doublet curve $C_{(\mathbf{3 , 2})_{1 / 6}}$ we consider line bundles $P_{(\mathbf{3 , 2})_{1 / 6}}$ which solve Eq. (4), where $\bar{K}_{B_{3}}{ }^{3}$ is the triple intersection number of the anticanonical class of the threefold $B_{3}$. This constraint has $N_{P}\left(C_{(\mathbf{3 , 2})_{1 / 6}}\right)=\left(2 \bar{K}_{B_{3}}{ }^{3}\right)^{2 g}$ solutions, where $g$ is the genus of $C_{(\mathbf{3 , 2})_{1 / 6}}$. In every QSM vacuum, the zero mode spectrum is counted by the cohomologies of one of these solutions. It is currently not known exactly which roots stem from F-theory gauge potentials in the Deligne cohomology $H_{D}^{4}\left(\hat{Y}_{4}, \mathbb{Z}(2)\right)$ of the elliptic fourfold $\hat{Y}_{4}$.

In this work, we did not attempt to give a detailed answer to this question. Rather, we focused on base spaces for which it can be expected that many roots are induced from inequivalent F-theory gauge potentials, i.e., gauge potentials which induce the same chiral index but differ in their $C_{3}$-flat directions. In the fourfold geometry $\hat{Y}_{4}$, these $C_{3}$-flat directions are described by the intermediate Jacobian $J^{2}\left(\hat{Y}_{4}\right)$. Since $h^{3,0}\left(\hat{Y}_{4}\right)=0$, it holds $J^{2}\left(\hat{Y}_{4}\right)=H^{2,1}\left(\hat{Y}_{4}\right) / H^{3}\left(\hat{Y}_{4}, \mathbb{Z}\right)$ (see [32] for details) and $\operatorname{dim}_{\mathbb{C}}\left(J^{2}\left(\hat{Y}_{4}\right)\right)=h^{2,1}\left(\hat{Y}_{4}\right)$. If a gauge potential in $H_{D}^{4}\left(\hat{Y}_{4}, \mathbb{Z}(2)\right)$ admits $\left(2 \bar{K}_{B_{3}}{ }^{3}\right)$ th roots, then it admits $\left(2 \bar{K}_{B_{3}}{ }^{3}\right)^{2 h^{2,1}\left(\hat{Y}_{4}\right)}$ roots. On $C_{(\mathbf{3}, 2)_{1 / 6}}$, we find $\left(2 \bar{K}_{B_{3}}\right)^{2 g}$ roots. In general, only a subset of these root bundles are physical. As elaborated on in the main text, we focus on geometries with $h^{2,1}\left(\hat{Y}_{4}\right) \geq g$. Statistically speaking, it is then likely that many, maybe even all, of the $\left(2 \bar{K}_{X_{\Sigma(T)}}^{3}\right)^{2 g}$ root bundles are physical.

The QSM toric base threefolds are obtained from FRSTs of 708 three-dimensional, reflexive, lattice polytopes [31]. In these spaces, the gauge divisors are K3 surfaces. This leads to gauge coupling unification and emphasizes the physical significance of $\bar{K}_{B_{3}}$. Only 37 of the QSM polytopes lead to spaces with $h^{2,1}\left(\hat{Y}_{4}\right) \geq g$. Still, the triangulations of these 37 polytopes provide the majority of the $\mathcal{O}\left(10^{15}\right)$ toric QSM base threefolds [31,49].

The natural next step is to count the number $N_{P}^{(3)}$ of roots which solve Eq. (4) and admit exactly three global sections, thus ensuring no vectorlike exotics on the quark-doublet curve. In following [41], we achieve this by studying limit roots on a nodal curve $C_{(\mathbf{3 , 2})_{1 / 6}}$ introduced in [41], which establishes a lower bound $\check{N}_{P}^{(3)} \leq N_{P}^{(3)}$. Crucially, we argue that $\check{N}_{P}^{(3)}$ is identical for all spaces $B_{3}\left(\Delta^{\circ}\right)$ obtained from FRSTs of $\Delta^{\circ}$, that is, $\breve{N}_{P}^{(3)}$ depends only on $\Delta^{\circ}$ and not the FRSTs.

We establish this result by arguing that the data, which specifies the limit roots, is identical for all spaces in $B_{3}\left(\Delta^{\circ}\right)$. This in turn follows by noting that the QSM base spaces are obtained from desingularizations of toric K3 hypersurfaces. Since the nodal curve $C_{(3,2)_{1 / 6}}^{\cdot}$ is closely related to the Picard lattice of the resulting smooth, toric K3 surface, we could employ powerful and well-known results about such desingularizations [44-47] (see also [48] for recent work on related topics), and thereby establish the claim. Explicitly, this reduces to the FRST invariance of topological triple-intersection numbers, which are related to FRST-independent counts of lattice points in the polytope $\Delta[45]$.

Among the 37 polytopes with $h^{21}\left(\hat{Y}_{4}\right) \geq g$, there are four polytopes for which $C_{(\mathbf{3 , 2})_{1 / 6}}$ has components with genus larger than one, so that the techniques introduced in [41] cannot be applied. For the remaining 33 polytopes, we list the lower bounds in Table I. These counts were determined with the Gap4 package QSMExplorer, which is part of the ToricVarieties_project [37]. We have optimized the input for this algorithm by simplifying the dual graph of $C_{(\mathbf{3 , 2})_{1 / 6}}$. For one polytope and a personal computer, we expect run times from a few seconds to around 10 minutes for the lower bounds in Table I.

Surprisingly, the simplifications of the dual graph of $C_{(\mathbf{3 , 2})_{1 / 6}}^{\cdot}$ lead to very similar graphs for distinct polytopes, and at times even identical lower bounds. For example, this applies to $\Delta_{128}^{\circ}, \Delta_{130}^{\circ}, \Delta_{136}^{\circ}$ and $\Delta_{236}^{\circ}$. We reserve a detailed study of this phenomenon for future work.

We read off from Table I that $\check{N}_{P}^{(3)} / N_{P}$ and $h^{21}\left(\hat{Y}_{4}\right) /$ $g\left(C_{(\mathbf{3 , 2})_{1 / 6}}\right)$ are largest for $B_{3}\left(\Delta_{8}^{\circ}\right)$. At least every 3000th root on $C_{(\mathbf{3 , 2})_{1 / 6}}$ has exactly three global sections. Furthermore, $\quad 16=h^{21}\left(\hat{Y}_{4}\right) \geq g \equiv g\left(C_{(\mathbf{3 , 2})_{1 / 6}}\right)=4, \quad$ for these spaces, which is the largest, respectively, smallest possible value among all QSMs. Therefore, the triangulations of $\Delta_{8}^{\circ}$ lead to $\mathcal{O}\left(10^{15}\right)$ [49] promising toric base threefolds for F-theory Standard Models with exactly three quark doublets and no vectorlike exotics in this representation.

The study of root bundles on the matter curves $C_{(\mathbf{1 , 1})_{1}}$ and $C_{(\mathbf{3}, \mathbf{1})_{-2 / 3}}$ is identical to the presented study of roots on $C_{(\mathbf{3 , 2})_{1 / 6}}$. The matter curve $C_{(\mathbf{3 , 1})_{1 / 3}}$ is more complicated due to its higher genus, but can at least, in principle, be treated analogously. The real challenge however, is to establish one vectorlike pair on the Higgs curve $C_{(\mathbf{1 , 2})_{-1 / 2}}$ and to investigate the top-down origin of the root bundles from F-theory gauge potentials. It can be anticipated that a detailed study of these questions will shed more light on the structure and construction of F-theory MSSMs. We hope to return to this thrilling and challenging task in the near future.

\section{ACKNOWLEDGMENTS}

We are grateful to Ron Donagi and Marielle Ong for past collaboration, insightful discussions and ongoing work on vectorlike spectra on the Higgs curve. The reliable computations of plesken.mathematik.unisiegen.de are truly appreciated. We thank Kamal 
Saleh and Jiahua Tian for valuable discussions. M. B is partially supported by NSF Grant No. DMS 201673 and by the Simons Foundation Collaboration Grant No. 390287 on "Homological Mirror Symmetry." The work of M. C. and M. L. is supported by DOE Award No. DE-SC013528Y. M. B. and M.C. further acknowledge support by the
Simons Foundation Collaboration Grant No. 724069 on "Special Holonomy in Geometry, Analysis and Physics." M. C. thanks the Slovenian Research Agency No. P1-0306 and the Fay R. and Eugene L. Langberg Chair for their support.
[1] P. Candelas, G. T. Horowitz, A. Strominger, and E. Witten, Nucl. Phys. B258, 46 (1985).

[2] B. R. Greene, K. H. Kirklin, P. J. Miron, and G. G. Ross, Phys. Lett. B 180, 69 (1986).

[3] V. Braun, Y.-H. He, B. A. Ovrut, and T. Pantev, Phys. Lett. B 618, 252 (2005).

[4] V. Bouchard and R. Donagi, Phys. Lett. B 633, 783 (2006).

[5] V. Bouchard, M. Cvetič, and R. Donagi, Nucl. Phys. B745, 62 (2006).

[6] L. B. Anderson, J. Gray, Y.-H. He, and A. Lukas, J. High Energy Phys. 02 (2010) 054.

[7] L. B. Anderson, J. Gray, A. Lukas, and E. Palti, Phys. Rev. D 84, 106005 (2011).

[8] L. B. Anderson, J. Gray, A. Lukas, and E. Palti, J. High Energy Phys. 06 (2012) 113.

[9] M. Berkooz, M. R. Douglas, and R. G. Leigh, Nucl. Phys. B480, 265 (1996).

[10] G. Aldazabal, S. Franco, L. E. Ibanez, R. Rabadan, and A. M. Uranga, J. Math. Phys. (N.Y.) 42, 3103 (2001).

[11] G. Aldazabal, S. Franco, L. E. Ibanez, R. Rabadan, and A. M. Uranga, J. High Energy Phys. 02 (2001) 047.

[12] L. E. Ibanez, F. Marchesano, and R. Rabadan, J. High Energy Phys. 11 (2001) 002.

[13] R. Blumenhagen, B. Kors, D. Lüst, and T. Ott, Nucl. Phys. B616, 3 (2001).

[14] M. Cvetič, G. Shiu, and A. M. Uranga, Phys. Rev. Lett. 87, 201801 (2001).

[15] M. Cvetič, G. Shiu, and A. M. Uranga, Nucl. Phys. B615, 3 (2001).

[16] R. Blumenhagen, M. Cvetič, P. Langacker, and G. Shiu, Annu. Rev. Nucl. Part. Sci. 55, 71 (2005).

[17] T. L. Gomez, S. Lukic, and I. Sols, Commun. Math. Phys. 276, 1 (2007).

[18] V. Bouchard and R. Donagi, J. High Energy Phys. 08 (2008) 060.

[19] C. Vafa, Nucl. Phys. B469, 403 (1996).

[20] D. R. Morrison and C. Vafa, Nucl. Phys. B473, 74 (1996).

[21] D. R. Morrison and C. Vafa, Nucl. Phys. B476, 437 (1996).

[22] T. W. Grimm and H. Hayashi, J. High Energy Phys. 03 (2012) 027.

[23] S. Krause, C. Mayrhofer, and T. Weigand, J. High Energy Phys. 08 (2012) 119.
[24] V. Braun, T. W. Grimm, and J. Keitel, J. High Energy Phys. 12 (2013) 069.

[25] M. Cvetič, A. Grassi, D. Klevers, and H. Piragua, J. High Energy Phys. 04 (2014) 010.

[26] M. Cvetič, D. Klevers, D. K. M. Peña, P.-K. Oehlmann, and J. Reuter, J. High Energy Phys. 08 (2015) 087.

[27] L. Lin, C. Mayrhofer, O. Till, and T. Weigand, J. High Energy Phys. 01 (2016) 098.

[28] L. Lin and T. Weigand, Nucl. Phys. B913, 209 (2016).

[29] S. Krause, C. Mayrhofer, and T. Weigand, Nucl. Phys. B858, 1 (2012).

[30] M. Cvetič, L. Lin, M. Liu, and P.-K. Oehlmann, J. High Energy Phys. 09 (2018) 089.

[31] M. Cvetič, J. Halverson, L. Lin, M. Liu, and J. Tian, Phys. Rev. Lett. 123, 101601 (2019).

[32] M. Bies, C. Mayrhofer, C. Pehle, and T. Weigand, arXiv:1402.5144.

[33] M. Bies, C. Mayrhofer, and T. Weigand, J. High Energy Phys. 11 (2017) 081.

[34] M. Bies, Cohomologies of coherent sheaves and massless spectra in F-theory, Ph.D. thesis, Heidelberg U. (2018-02).

[35] M. Bies, M. Cvetič, R. Donagi, L. Lin, M. Liu, and F. Ruehle, J. High Energy Phys. 01 (2021) 196.

[36] M. Bies, M. Cvetič, R. Donagi, L. Lin, M. Liu, and F. Ruehle, Database, https://github.com/Learning-line-bundlecohomology, 2020.

[37] The ToricVarieties_project authors, The ToricVarieties_ project, https://github.com/homalg-project/ToricVarieties_ project, 2019-2021.

[38] A. Brill and M. Nöther, Math. Ann. 7, 269 (1874).

[39] D. Eisenbud, M. Green, and J. Harris, Bull. Am. Math. Soc. 33, 295 (1996).

[40] T. Watari, Phys. Lett. B 762, 145 (2016).

[41] M. Bies, M. Cvetič, R. Donagi, M. Liu, and M. Ong, arXiv:2102.10115.

[42] C. Lucia, C. Cinzia, and C. Maurizio, Trans. Am. Math. Soc. 359, 3733 (2007).

[43] M. Kreuzer and H. Skarke, Adv. Theor. Math. Phys. 2, 853 (1998).

[44] V. V. Batyrev, J. Alg. Geom. 3, 493 (1994).

[45] E. Perevalov and H. Skarke, Nucl. Phys. B505, 679 (1997).

[46] D. A. Cox and S. Katz, Mirror Symmetry and Algebraic Geometry, Mathematical surveys and monographs (American Mathematical Society, Providence, 1999). 
[47] F. Rohsiepe, arXiv:hep-th/0409290.

[48] A. P. Braun, C. Long, L. McAllister, M. Stillman, and B. Sung, arXiv:1712.04946.

[49] J. Halverson and J. Tian, Phys. Rev. D 95, 026005 (2017).
[50] D. Cox, J. Little, and H. Schenck, Toric Varieties, Graduate studies in mathematics (American Mathematical Society, Providence, 2011).

[51] M. Kreuzer, Ukr. J. Phys. 55, 613 (2010). 